\title{
Acquisition and extinction, within subjects, of a continuously rewarded response and a response learned under discontinuous negatively correlated reward'
}

MICHAEL E. RASHOTTE AND ABRAM AMSEL UNIVERSITY OF TORONTO

Some data are presented from an experimental situation in which the same $S$ runs to continuous reward in the presence of one stimulus and to Logan's discontinuous negatively correlated reward to another stimulus. Differences in performance to the two stimuli are in terms of average speeds and profiles of the animal's performance across a fractionated alley.

The purpose of this paper is to report some preliminary data obtained from an experiment in which rats were trained, concurrently, in one runway under a condition of discontinuous, negatively correlated (DNC) reward and in another under continuous reward (CRF).

Presented by Logan (1961) as a discrete-trial analogue of the free-operant DRL reward schedule (see Ferster \& Skinner, 1957), the DNC reward condition has been employed in a number of experiments (Logan, 1960 , ch. 8) primarily to accumulate evidence favorable to a micromolar analysis of responses (Logan, $1956,1960)$. The critical feature of this condition of reward is that, to be rewarded, $S$ must take $x$ or more seconds to complete a given approach response; should $S$ take fewer than $x$ seconds, reward is withheld.

Data from such experiments show that on a high percentage of trials Ss can successfully consume the appropriate number of seconds and obtain reward, and that, in the process, Ss show idiosyncratic patterns of behaviors in the runway which compete with the approach response and reduce running speed.

The purposes of the present experiment were (a) to see whether these characteristics of performance under DNC would appear if Ss were receiving CRF in another runway within the same phase of the experiment, and (b) to make comparisons between performance in the two runways during acquisition and extinction.

Method

The apparatus (described in detail by Amsel, Rashotte, \& MacKinnon, 1966) consisted of two straight runways, $3-3 / 4$ in high, $2-7 / 8$ in. wide and 63 in. long, one painted flat black and the other flat white. Either runway was entered through a common entrybox-start-box unit, painted flat gray. The final 14 in. of the runway, enclosed by a guillotine door, served as a goal box. A system of photocells and silent clocks allowed measures of time to traverse the five successive 1-ft. segments of the runway after the start door had opened. On rewarded trials a Gerbrands feeder dispensed a 250-mg Noyes pellet when $S$ broke the fifth photobeam positioned $5 \mathrm{ft}$. from the start door and 3 in. from the food cup. On nonrewarded trials, and during extinction, the feeder was not activated.

Five male albino rats, about 140 days old at the beginning of training, were maintained on a $10 \mathrm{gm}-$ $23 \mathrm{hr}$. food deprivation regimen for five weeks preceding the beginning of the experiment. Each $S$ received eight trials per day, four in the black runway and four in the white, in a quasi-random order, for 36 acquisition days and for at least five extinction days. For two Ss the white runway signalled the DNC reward condition and the black runway the CRF condition; for the remaining three Ss the color-reward relationship was reversed. During acquisition Ss were rewarded in the DNC runway only when the time to reach the fifth photobeam, counting from the raising of the start door, equalled or exceeded $5 \mathrm{sec}$. Reward was always delivered in the CRF runway whenever $S$ broke the last photobeam. Ss were removed from the apparatus as soon as they had finished eating or, on nonrewarded trials, after $30 \mathrm{sec}$. Ss were run in a different order on each day and the intertrial interval was approximately $5 \mathrm{~min}$.

\section{Results and Discussion}

There were only minor differences in performance between the color-control Ss. The median speed for all Ss over the entire length of the runway is presented for each runway in Fig. 1. Clearly, Ss learned to run slowly in the DNC runway and more rapidly in the CRF runway in acquisition. The terminal speed in the DNC runway was about $1.25 \mathrm{ft} . / \mathrm{sec}$., slightly higher than the $1 \mathrm{ft} . / \mathrm{sec}$, required by the reward condition. The percentage of rewards earned in the DNC runway over the last 12 days of acquisition also reflects less than perfect adjustment to the requirements of the condition at the time acquisition was terminated. For the five Ss these were 25, 50,6, 21 and 50, somewhat lower terminal percentages than those reported by Logan $(1960$, ch. 8 ) in experiments involving DNC training only, although Logan ran many more trials than we did. The extinction data in Fig. 1 show that 


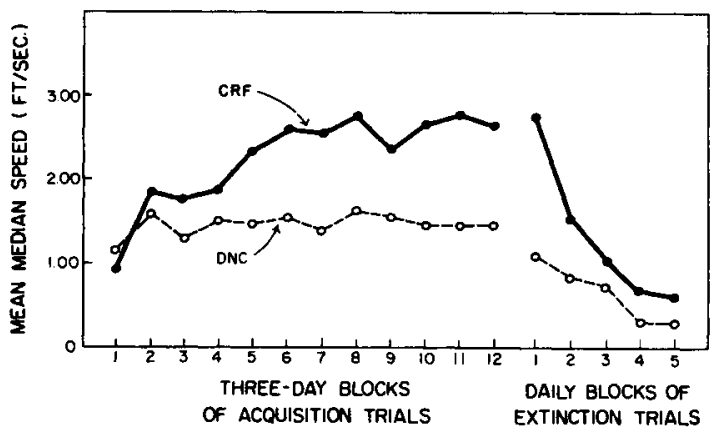

Fig. 1. Mean median speed in DNC and CRF runways in acquisition (3-day blocks) and extinction (dailv blocks).

performance in both DNC and CRF alleys drops well below acquisition level.

Each rat appeared to develop a pattern of idiosyncratic ("superstitious") behavior in the DNC runway in the process of meeting the DNC requirement. One way to look at these behaviors objectively and quantitatively, and to highlight the difference between performance in the DNC and CRF runways, is to fractionate the total runway response into five $1-\mathrm{ft}$. segments and plot response speed across these segments for the DNC and CRF trials. This can be done for each trial or for blocks of trials for each $S$ and the resulting curves constitute profiles of each S's performance to the two stimuli. Such profiles are shown for one $S$ in Fig. 2 for the even-numbered acquisition days and for each of the first four and last extinction days. In the DNC alley, this $S$ ran relatively rapidly up to the fourth alley segment where it stopped before dashing into the goal area. Pausing in the fourth segment came to be accompanied by a ritualized movement of the head, from side to side across the alley. In the CRF alley the approach response took the form

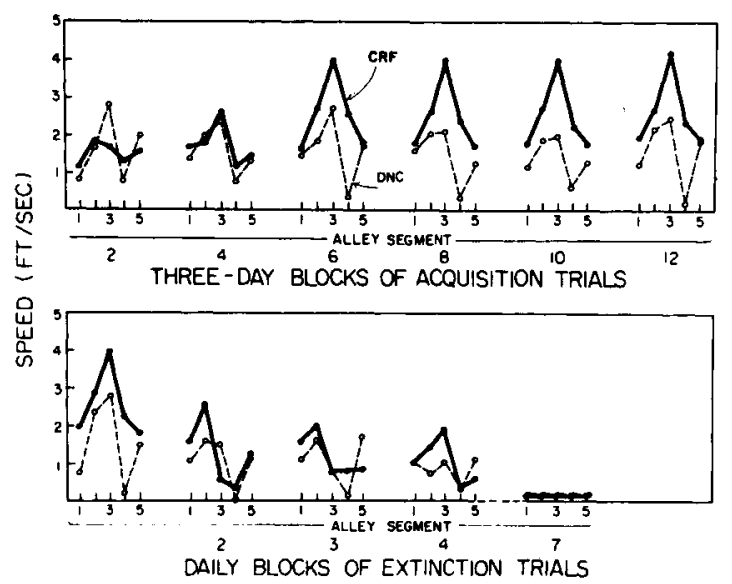

Fig. 2. Acquisition and extinction response profiles in DNC and CRF runways for one $S$. of uninterrupted running, with fastest speeds in the middle segment. A similar CRF profile was found in four of the five Ss in this experiment.

In extinction the first-day profiles are like the terminal acquisition ones; however, on Days 2, 3 and 4 of extinction, the ritualized head-moving response appeared in the CRF runway in, or near, the fourth alley segment and the CRF response profile changed to approximate the DNC profile. Other Ss, which had developed different behaviors in the DNC runway (such as biting at the start door), showed these behaviors during extinction in the CRF runway, although the conformity of their CRF to their DNC profile was not as great as that in Fig. 2.

This extinction finding could be construed as further support for an interpretation of the generalized partial reinforcement effect (Brown \& Logan, 1965) in within-S PRF experiments which is based on mediating properties of anticipatory frustration $\left(r_{F^{-s}}\right.$ ) (Amsel, 1967; Amsel, Rashotte, \& MacKinnon, 1966). It could be argued that, for each $S$, the low percentage reward in the DNC runway promoted conditioning of $s_{F}$ to a specific form of the DNC approach response Rashotte, 1966), and that, in extinction, $r_{F}^{-s_{F}}$ was evoked in the CRF runway and acted to elicit that form of the approach response to which it had earlier been conditioned.

Several further investigations have verified the present findings and will be reported in more detail and with a fuller discussion at a later date.

\section{References}

Amsel, A. Partial reinforcement effects on vigor and persistence: Advances in frustration theory derived from a variety of withinsubjects experiments. In K. W. Spence \& J. T. Spence (Eds.), The psychology of learning and motivation. New York: Academic Press (in press).

Amsel, A., Rashotte, M. E., \& Mackinnon, J. R. Partial reinforcement effects within subjects and between subjects. Psychol. Monogr., 1966, 80, No, 20 (Whole No. 628).

Brown, R. T., \& Logan, F. A. Generalized partial reinforcement effect. J. comp. physiol. Psychol., 1965, 60, 64-69.

Ferster, C. B., \& Skinner, B. F. Schedules of reinforcement. New York: Appleton-Century-Crofts, 1957.

Logan, F. A. A micromolar approach to behavior theory. Psychol. Rev., 1956, 63, 63-73.

Logan, F. A. Incentive: How the conditions of reinforcement affect the performance of rats. New Haven: Yale University Press, 1960.

Logan, F. A. Discrete-trials DRL. J. exp. Anal. Behav., 1961, 4, 277-279.

Rashotte, M. E. Frustrative factors in persistence: Within- and between-subject comparisons. Unpublished doctoral dissertation. University of Toronto, 1966

\section{Note}

1. This research was supported by grants from the National Research Council of Canada (APB-72) and the National Science Foundation (GB-3772) to Abram Amsel. The authors wish to thank Gail Dichmann and Joanna Boehnert who helped collect a part of the data. 